\title{
Surgical anatomy of the aortic valve and root-implications for valve repair
}

\author{
Ruggero De Paulis, Andrea Salica \\ Department of Cardiac Surgery, European Hospital, Rome, Italy \\ Correspondence to: Ruggero De Paulis. Department of Cardiac Surgery, European Hospital, Via Portuense 700, 00149 Rome, Italy. \\ Email: rdepaulis58@gmail.com.
}

\begin{abstract}
The aortic root is an important anatomical structure positioned at the center of the heart, making it critical to the functioning of the major cardiac chambers. Deep knowledge of the anatomical "surroundings" of the aortic root is crucial for surgeon attempting to spare or repair a leaking aortic valve. In fact, root dissection is a necessary step to "skeletonize" the aortic valve, allowing the surgeon to work on the critical components of its structure, namely the aorto-ventricular junction, the virtual basal ring (VBR) and the sino-tubular junction (STJ). These three components, along with the insertion of the leaflet to the aortic wall, form the skeleton of the aortic valve that is essential in guaranteeing valve competence. A good anatomical proportion between the various component of the skeleton of the aortic valve need to be verified, or re-established in order to set the basis for an optimal aortic valve repair. Once the skeleton of the heart has been correctly addressed, the condition of the valve leaflets need to be considered. Excess of leaflet tissue is treated by leaflet plication or resection and lack of leaflet tissue is addressed by tissue extension with autologous or heterologous materials. In the present manuscript, we highlight the principal structure of the aortic root and describe in detail each anatomical component. This basic anatomical knowledge is also important for a through understanding of the normal function of the valve and root structure during the cardiac cycle. The close boundaries existing between the left ventricular cavity and the aorta are important in explaining the sophisticated function of opening and closing of the aortic valve. Similarly, the role played by the sinuses of Valsalva in regulating the blood flow exiting the ventricle underline the concept that "form follows function" and emphasizes the importance of a good anatomical reconstruction for an optimal and long-lasting valve function.
\end{abstract}

Keywords: Aortic root; aortic valve; valve sparing; valve repair; anatomy

Submitted Mar 01, 2019. Accepted for publication Apr 27, 2019.

doi: 10.21037 /acs.2019.04.16

View this article at: http://dx.doi.org/10.21037/acs.2019.04.16

\section{General considerations}

The aortic root is considered the "centerpiece" of the heart like a bridge between the left ventricle and the ascending aorta. From its inner and outer surface, the aortic root has relationship to all cardiac chambers, the left ventricle, the left atrium, the right ventricle, and the right atrium (1). The accurate knowledge of its peculiar anatomy is fundamental in approaching aortic root surgery in general and aortic valve-sparing and/or repair in particular. The aortic root encompasses the aortic valve, gives insertion to the base of the anterior leaflet of the mitral valve, and gives origin to the coronary arteries. Besides its main anatomical function, the aortic root has a part in the process of the aortic leaflets interaction with the sinuses of Valsalva and in the regulation of blood flow dynamics during the complete cardiac cycle $(2,3)$. Despite the aortic root being well-defined anatomically, there are still controversies on the best way to describe it in terms of its separate components, with specific reference to the aortic annulus (4).

The aortic annulus has more than one definition. Surgeons commonly consider the aortic annulus as the place where the cusp are attached to the aortic wall and anatomically exemplify the division between the left 


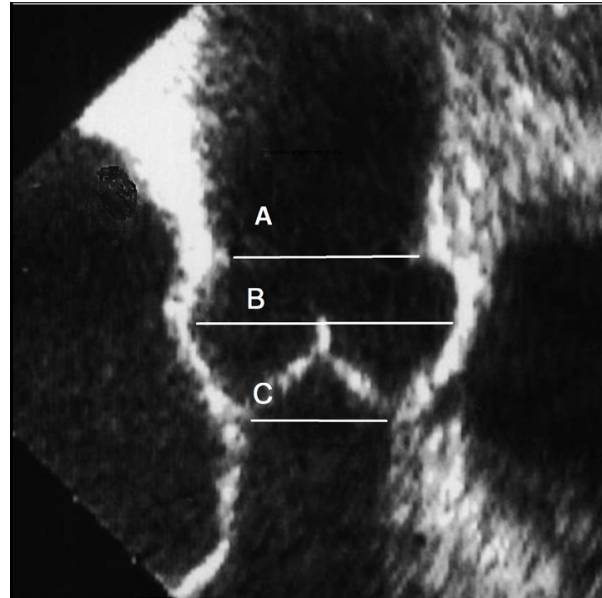

Figure 1 This is an echocardiographic representation of the aortic root in a mid-esophageal $120^{\circ}$ view. In the figure the three main diameters of the root are evidenced. A: diameter of the STJ, measured at the level of the narrowing between the root and the tubular segment of the ascending aorta; B: the diameter of the Sinuses of Valsalva, measured at the level of the maximum bulging of the sinuses; C: the diameter of the virtual basal ring, measured by joining the nadir of the leaflets (this is the echocardiographic parameter considered to provide measurement of the diameter of the aortic annulus). The echocardiographic diameter of the STJ in a normal healthy heart, is approximately $75 \%$ of the maximal sinus diameter, and is larger than aortic annulus at the level of the VBR with a ratio of 1.3 . VBR, virtual basal ring; STJ, sino-tubular junction.

ventricle and the sinuses of Valsalva (5). In contrast, cardiologists or radiologists, consider the aortic annulus to be the virtual basal ring (VBR), which is formed by the nadir of each leaflet joining in a circular shape (Figure 1). These clear and simple definitions have been widely accepted. The aortic annulus however, has greater complexity and its' morphology is represented by a clear three-dimensional shape (Figure 2).

El Khoury and colleagues, identified the aortic annulus as a functional unit, introducing the concept of the functional aortic annulus (FAA). It is comprised of (I) the sino-tubular junction (STJ), (II) the semilunar attachments of the aortic leaflets and (III) the virtual line that joins each leaflet nadir and the respective inter-leaflet triangles, the VBR (6). In this way the aortic annulus could be considered the skeleton of the aortic root.

The basal attachments of the aortic leaflets describe three semilunar lines with a distinctive crown-like formation.

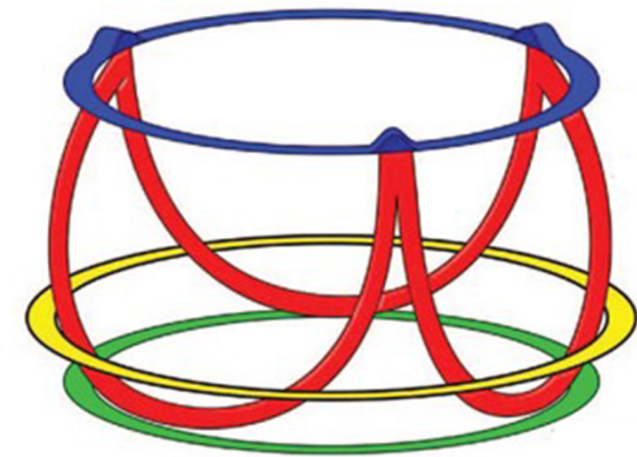

Figure 2 This is a well-known representation of the so called Functional Aortic Annulus. It appears as a tridimensional structure involving the entire aortic root all along its extension. It is composed by the sino-tubular junction (blue line), that represents the highest part of the aortic annulus and a boundary line between the root and the tubular tract of the ascending aorta; the aortic leaflets attachment (red line), characterized by a crown-like shape starting from the sino-tubular junction, where forms the three commissures, till to the upper portion of the left ventricle outflow tract. It is considered the hemodynamic annulus because it represent the boundaries between ventricular pressure and aortic pressure; the VAJ (the yellow line), that is an almost circular line across the lower third of the aortic leaflets and have a specific anatomical counterpart constituted by muscular crescents of the interventricular septum, the membranous septum and the mitroaortic curtain; the virtual basal ring (green line), that is a functional line formed by joining the nadir of the three leaflet, and is commonly considered the echocardiographic aortic annulus. VAJ, ventriculo-aortic junction.

The three leaflets attachments represent the hemodynamic boundary line that separates the left ventricular outflow tract (LVOT) from the arterial system. Each aortic leaflet, due to its semilunar line, is characterized by a nadir positioned in the most distal portion of the LVOT and a zenith that rises and joins the STJ. It is noteworthy that the STJ, known also as the supra-aortic ridge, is the upper part of the aortic annulus. This hemodynamic annulus, represented by the attachments of the leaflets, is definitely different from the anatomical boundary line that separate the LVOT from the arterial system. This anatomical boundary is in fact evident in the ventriculo-aortic junction (VAJ), the real anatomical locus where the LVOT join the aortic tissues.

The anatomical continuity between STJ, commissures, inter-leaflets triangles, leaflets attachments, VAJ and VBR is fundamental to understand the strong interaction between 


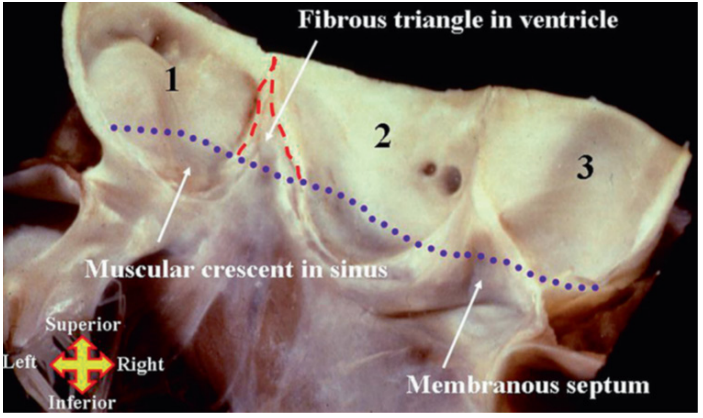

Figure 3 This is an anatomical view of the ventriculo-aortic junction and of its anatomical counterpart composed by muscular fibers of the left ventricle, the membranous septum and the mitro-aortic curtain. It is the anatomical borderline between the left ventricle and the arterial system. Each leaflet has different relationships with the components of this structure. Left ventricular fibers embody the aortic root wall with muscular inclusions at the level of the right coronary sinus, left/right commissure and a part of the left coronary sinus towards the right sinus. The right/non coronary commissure takes relationship with the membranous septum, while the non-coronary sinus and the non/left coronary commissure have relationships with the mitroaortic curtain.

the anatomy and the physiology of the aortic valve. It is a guide to perform root surgery in general and to soundly approach aortic valve-sparing/repair surgery. In particular, it is fundamental to understand the importance and the role of the aortic annuloplasty and the differences between the remodeling and reimplantation techniques.

\section{The STJ}

The STJ joins upwards the tubular portion of the aorta and downward the sinuses of Valsalva and the aortic commissure with which it is in direct continuity. The STJ, on the aortic lumen, commonly presents as a marginally elevated ridge of thickened aortic wall. Its' appearance on the outside is smooth and is usually identifiable after a dissection of the upper portion of the aortic root. The STJ is not perfectly circular and displays a mildly trefoil or scalloped outline.

The STJ plays an essential role in the aortic root architecture and the aortic valve function, as part of the FAA (6). It has a specific geometric relation with the other parts of the root. Despite the area of the STJ increasing with age and hypertensive cardiomyopathy (7), a normal healthy heart has STJ diameters of approximately $75 \%$ of the maximal sinus diameter (8). While at the level of the VBR it is larger than the aortic annulus with a ratio of 1.3 (Figure 1). A mismatch in this ratio between the STJ and the VBR is a frequent cause of aortic regurgitation. Re-establishing the correct proportion between the VBR and the STJ is a fundamental step when approaching any procedure of valve-sparing or valve repair.

\section{The VAJ and the VBR}

The VAJ represents an anatomical site where ventricular structures including muscular septum, mitro-aortic curtain and membranous septum, join the arterial system. It is positioned into the wall of the aorta. Diagrammatically, the VAJ is characterized by a relatively circular line created by joining the base of the inter-leaflet triangles with the lower third of the sinuses of Valsalva, slightly above the nadir of the aortic leaflets crossing in several points the semilunar line of the aortic leaflets (Figure 3) $(9,10)$.

Each sinus of the VAJ is characterized by distinctive differences. The three leaflets each have varying relationships with the mitro-aortic junction, ventricular muscle and the membranous septum. Left ventricular fibers embody the aortic root wall with muscular inclusions at the level of the right coronary sinus (RCS), left/right commissure and a section of the left coronary sinus (LCS) towards the right sinus. The right/non-coronary commissure has a relationship with the membranous septum, while the non-coronary sinus (NCS) and the non/ left coronary commissure have relationships with the mitroaortic curtain. It is because of these relationships that the VAJ seems an almost circular line, with a slight threedimensional morphology. Of note, is that the VAJ is further away at the level of the RCS and closer at the level of the NCS with respect to the VBR.

From a histologic point of view, the VAJ has variable thickness along the circumference of the aortic root. This is due to muscular inclusions being present and thickening the respective portion of the root. It is true that the left/right commissure and the RCS are significantly thicker (maximum thickness at the level of the RCS with $4.6 \mathrm{~mm}$ ) when compared with the right/non commissure, NCS, left/non coronary commissure and LCS where muscular inclusion of the left ventricle give way to the membranous septum and mitro-aortic curtain, with a mean thickness lower than $1 \mathrm{~mm}$ (Figure 4). Therefore, the total mean thickness of the aortic root is approximately $3 \mathrm{~mm}$ (11).

It is significant to underline the very relevant differences 

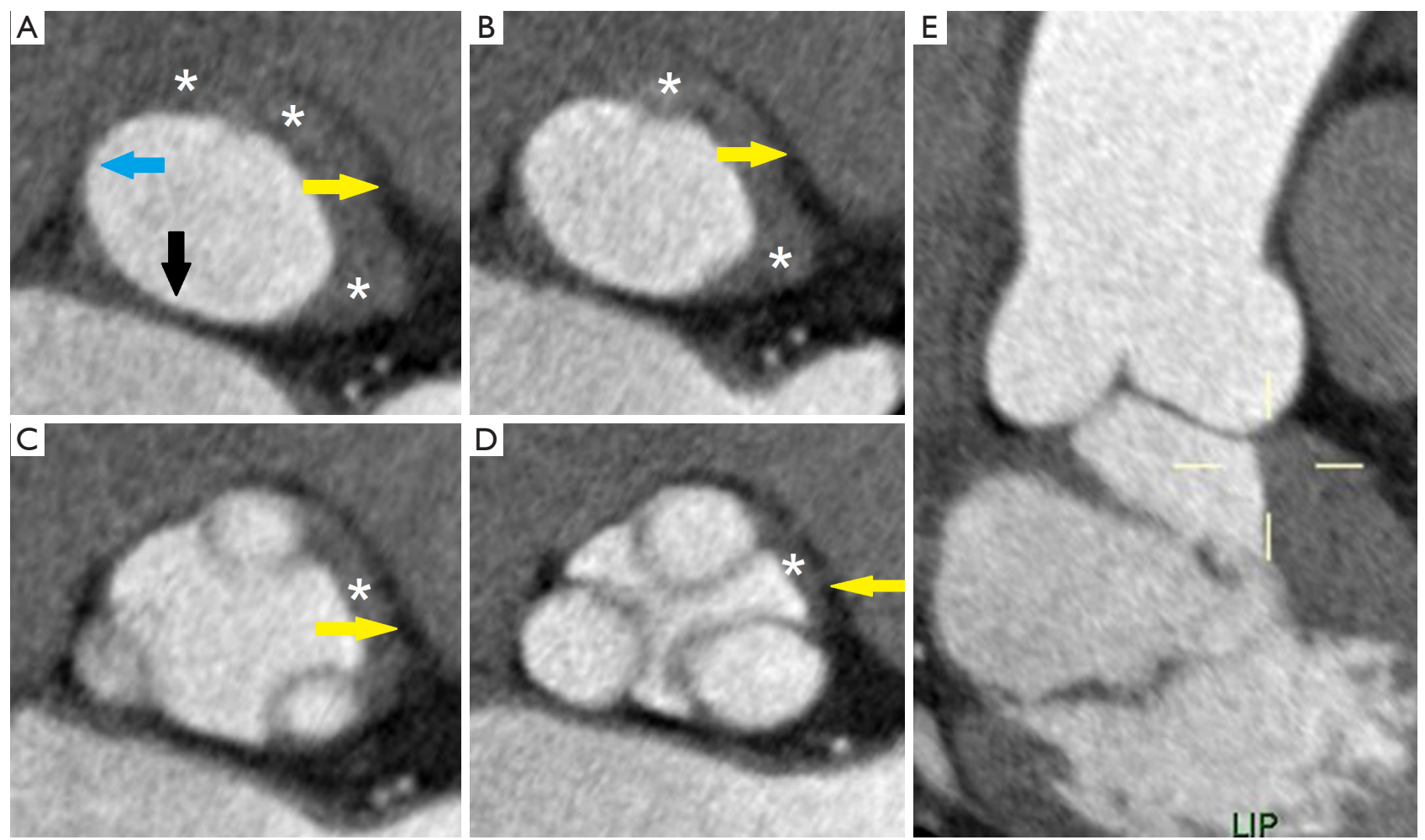

Figure 4 This picture evidences the anatomical relationships between the Virtual Basal Ring and the Ventriculo-aortic Junction by angio CT scan view. In (A) it is represented the virtual basal ring with its elliptical shape. It is well visible the muscular aspect of the left ventricle (white asterisks), the mitro-aortic curtain (black arrow) and the membranous septum (blue arrows). It is important to point out the presence of a cleavage plane between the muscular component of the left ventricle and the infundibulum of the right ventricle (yellow arrows). This boundary line is visible all along the extension of the external face of the root when moving more cranially (A,B,C,D, yellow arrows). This is the anatomical plane that permits externally to reach the level of the virtual basal ring despite the presence of muscular fibers as part of the ventriculo-aortic junction. From the histologic point of view, the thickness of the VAJ is variable along the aortic root circumference. The presence of muscular inclusions (white asterisks) thicken the respective portion of the root as evidenced in the (A,B,C,D). In fact, the left/right commissure and the right coronary sinus are significantly thicker than the right/non commissure, non coronary sinus, left/non coronary commissure and left coronary sinus where muscular inclusion of the left ventricle gives way to the membranous septum and mitroaortic curtain. (E) is a long axis view of the aortic root and ascending aorta. It is identifiable the insertion of the anterior mitral leaflet on ventriculo-aortic junction at the level of the non-coronary sinus and the muscular component at the level of the right coronary sinus. VAJ, ventriculo-aortic junction.

between the VAJ and the VBR. The VAJ is a real anatomic region defined by a specific histologic entity. The VBR represents a virtual circular line positioned inside the left ventricle outflow tract, running through the nadir of the aortic leaflets and the respective inter-leaflet triangles. It makes anatomical connections with the interventricular muscle septum, the membranous septum and the anterior mitral leaflet, but it is no more than a virtual plane, simply created connecting together the nadirs of the leaflets, without a real anatomic counterpart. Despite it having been considered a circular line, in vivo CT scan studies have accurately described its elliptical shape (Figure 4) $(12,13)$. It follows, therefore, that the measured diameters of the aortic annulus will vary if measured along its minor or major diameter. Bisecting and off-center cuts represent well identifiable lines, mainly used for accurate CT measurements of the root at the level of sinuses of Valsalva but are also utilized for a correct measurement of the aortic annulus. In fact, because the VBR is elliptical, measuring the VBR diameters using the bisecting cut has been proven to be more accurate while off-center cuts result in diameters significantly larger than those measured with the bisecting 

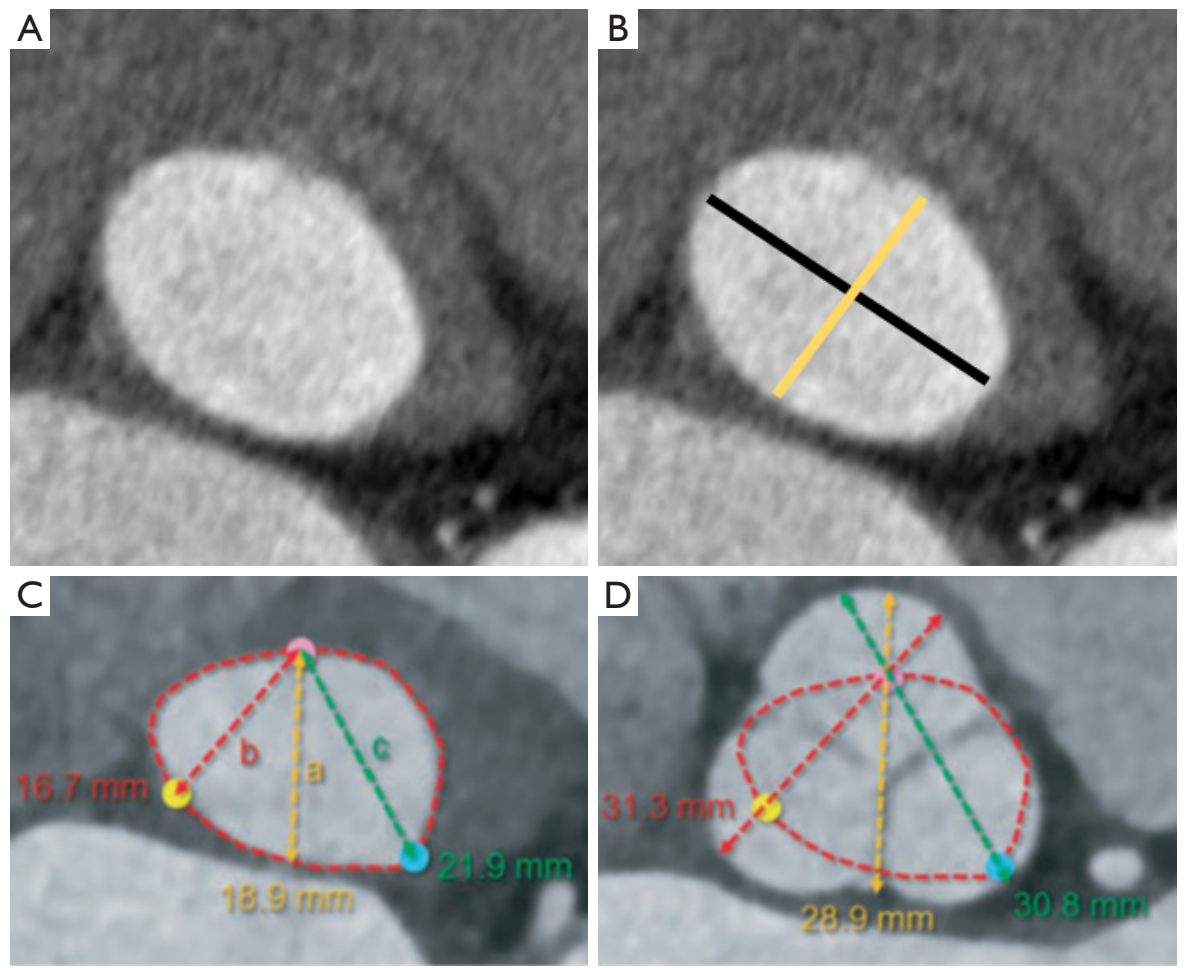

Figure 5 In this figure is represented the VBR in CT scan view. It is a virtual line positioned inside the left ventricle outflow tract, running through the nadir of the aortic leaflets. It takes anatomical relationships with the interventricular muscle septum, the membranous septum and the anterior mitral leaflet, but it is no more than a virtual plane, simply created connecting together the nadirs of the leaflets, without a real anatomic counterpart. Its elliptical shape has been well described by in vivo CT scan (A) with a major and a minor diameter (B). VBR is also measured using bisecting (yellow) and off-center nadir-to-nadir cuts (red and green) that represents well identifiable lines (C), because of their anatomical references with the sinuses of Valsalva (D). Measuring the VBR diameters using the bisecting cuts has been proved more accurate. In fact, because of the elliptical shape of the VBR, the off-center cuts result in diameters significantly larger than those measured with the bisecting cuts. VBR, virtual basal ring.

cut (14) (Figure 5).

In contrast to the right ventricle, with its free-standing infundibulum, where muscular fibers are found at the base of each pulmonary sinuses, the aortic VBR run across nonuniform components. It is fibrous tissue in the case of the mitro-aortic curtain and the membranous septum, or muscular under the right cusp. The hypothesis that these different types of tissues influence the elliptical shape of the aortic VBR, as observed in CT scan studies, appears suggestive (Figure 6).

\section{Aortic leaflets, commissures and inter-leaflets triangles}

The normal aortic valve is made up of three leaflets that serve as the moving parts of the valve. Each has a wrinkly surface that faces the aorta and a smoother surface that faces the ventricle. Each leaflet comprises a free margin that is slightly thicker than the basal portion and participates to valve closure during the diastole. The apposition zones, the so-called "lunulae", are on the ventricular surface, below the free margin representing the place where each leaflet meets the adjacent leaflets during aortic valve closure. At the midportion of the "lunulae", there is a further thickening called the "nodule of Arantius". Recognition of these anatomical parts, "lunulae" and "nodule of Arantius", is important to understand the physiopathology of valve regurgitation and the possibility of surgical repair. In fact, aortic regurgitation is mainly caused by the lack of apposition of these structures that can be caused either by tissue retraction or by enlargement of the skeleton of the root, mainly at the level of the annulus and/or ST junction. 


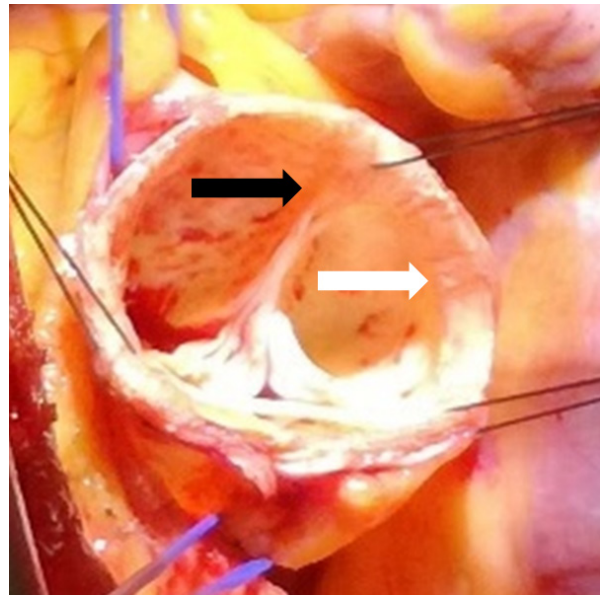

Figure 6 The basal margin of the leaflets is attached in a semilunar fashion to the aortic root. This basal attachment has the nadir below the VAJ and the zenith at the level of the STJ (white arrow), where each leaflet, joining the adjacent leaflet, form the three commissures respectively (black arrow). VAJ, ventriculo-aortic junction; STJ, sino-tubular junction.

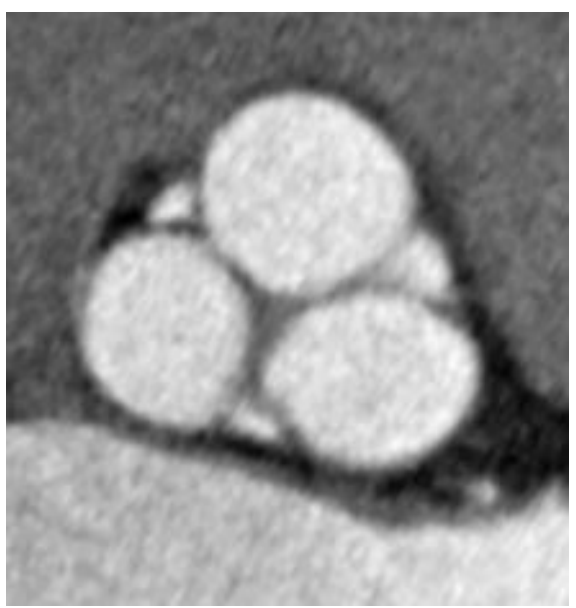

Figure 7 This CT scan view shows that beneath the apex of each commissures, there is a thin layer of fibrous tissue that goes down, between the respective leaflets, towards the VAJ and forms the final part the LVOT. These thinner areas of fibrous wall are described as the inter-leaflet triangles. For this reason, the crownlike line of the attachment of the leaflets divides the aortic root in sub-valvar and supra-valvar regions. The supra-valvular region, despite the presence of ventricular cells (at the level of the VAJ), is an aortic portion, while the sub-valvular region, despite the presence of aortic fibro-elastic cells (the interleaflet triangles) is a ventricular portion. Finally, the inter-leaflets triangles separate the inside of the left ventricle from the extra-cardiac space. LVOT, left ventricular outflow tract; VAJ, ventriculo-aortic junction.
The basal margin of the leaflets attach to the aortic root in a semilunar fashion. This basal attachment has the nadir below the VAJ and the zenith at the level of the STJ, where each leaflet, joining the adjacent leaflet, form the three commissures respectively. Underneath the apex of the commissures between the respective leaflets, there is a thin layer of fibrous tissue, in the shape of a triangle with its base towards the VAJ that forms the final part the LVOT. These thinner areas of fibrous wall are described as the inter-leaflet triangles. For this reason, the crownlike line of the attachment of the leaflets divides the aortic root in sub- and supra-valvular regions. The supra-valvular region, albeit containing ventricular cells (at the level of the VAJ), is an aortic portion, while the sub-valvular region, albeit containing aortic fibro-elastic cells (the interleaflet triangles) is a ventricular portion (10). Furthermore, the inter/leaflets triangles separate the inside of the left ventricle from the extra-cardiac space (Figure 7).

The total area of the leaflets when compared to the aortic root, is approximately $40 \%$ greater, with the largest area measured in the non-coronary leaflet and the smallest to the left coronary leaflet in most cases. This observation is fundamental in understanding the importance of aortic valve coaptation and the importance of the right proportion between overall root area and cusp surface.

\section{The sinuses of Valsalva}

The sinus of Valsalva is defined as the three-dimensional spaces of the aortic root surrounding the aortic leaflets. These sinuses represent the uppermost part of the arterial system proximal to the aortic valve. Cross-sectionally, the three bulges appear as a clover shape and due to dilated morphology, the root has significantly greater diameters at the midpoint of the sinuses than those measured at the sinotubular junction or at the basal attachment of the leaflets.

In theoretical terms, each of the three sinuses of the root are identical, noting that two of them give rise to the coronary arteries and are subsequently called the right and the LCSs. A portion of ventricular musculature, relative to the VAJ, is incorporated all along the base of the RCS and in the part of the LCSs close to the right sinus. The third sinus, known also as the non-coronary aortic sinus, has an exclusively arterial wall. Of note is that the basal part of this sinus is comprised of a portion of the mitro-aortic curtain. It is at this level the VAJ and the VBR coincide.

Although, the sinuses of Valsalva could be schematized 
as having a symmetrical pear-like presentation, its true morphology is more elaborate with several peculiarities and a somewhat asymmetrical shape. It is well accepted that for each sinus, there can be differences in both the internal height and volume. The LCS has consistently been found to be the smallest while the RCS was found to be similar to the NCS, which in many cases appears to be the tallest and largest of the three $(7,15,16)$. In fact, the aortic root naturally follows the curvature of the ascending aorta and it has been widely noted that there is the presence of a tilt angle of $5.5^{\circ}-11^{\circ}$ between the VBR and STJ planes $(7,15,16)$. Recreation of a sinuses-like space in root surgery mimics the physiology of the aortic valve.

In fact, a sinus-like space promotes the normal aortic leaflets opening and closing movements during the cardiac cycle. It is instrumental in the fluid-structure interaction between blood flow and aortic leaflets improving the systolic energy loss across the aortic valve and the diastolic coronary perfusion. Moreover, presence of the sinus-like space reduces the stress on the coronary button after their reimplantation (17-28).

\section{The aortic root and surgical considerations}

Previously in this paper, the principal components of the aortic root have been considered as single units so that their characteristics could be better understood. Now we will describe the aortic root in its integrity in order to understand the basis for a correct surgical approach. As much as the comprehension of the peculiarity of the VAJ and VBR have critical relevance in aortic valve repair surgery every time aortic annuloplasty or annular stabilization is performed, similarly, the anatomic relationship of the external face of the root with the contiguous cardiac structures have fundamental relevance for the dissection of the root needed to perform a reimplantation type of valve-sparing procedure. Analyzing every single anatomical structure along the circumferential plane, is of paramount importance when dissecting the root from its surrounding. The external wall of the right coronary sinus and the commissure between the right and the LCS, take close anatomical relationships with the right ventricle and the dissection should include the right ventricular muscle that needs to be separated from the aortic wall until the plane of dissection reaches the inviolable external boundary line represented by the VAJ. As we have seen, at this level the VAJ and the VBR are placed at different height; in fact, the VBR is placed at the nadir of the leaflet, while the VAJ comes above this plane.

Sutures passed at the level of the VBR, from the internal face of the root toward the external face (normally used to place an annuloplasty ring or to hold the graft in a reimplantation procedure), allows an affective annular stabilization despite this difference in height. Alternatively, part of the right ventricular wall needs to be dissected in order to go further down at the level of the VBR but the relative thickness of the wall (distance between the inner and outer points) will be increased. The external wall of the LCS, the commissure between the left and the NCS and of the NCS makes anatomical relationship with the roof of the left atrium. The separation of the soft connective tissue between the left atrial muscle and the aortic root allows an easier dissection down to the level of the VBR.

In fact, at this level the VAJ lacks ventricular fibers and coincides with the VBR. The commissure between the right and the NCS makes a relationship with the right atrium. At this level, the VAJ is represented by the membranous interventricular septum and the insertion of the tricuspid valve septal leaflet forms here an inviolable boundary line of the VAJ. The height of the internal root (measured from the VBR to the STJ) is usually greater than the corresponding external height (measured from the VAJ to the STJ). In this context, the measurements are significantly affected by the non-planar nature of the VAJ. On the side of the RCS, the external root height is significantly shorter when compared with the NCS and LCS (11). The presence of muscular inclusions, place the VAJ farther from the VBR and nearer to the STJ at this level, while the absence of muscular inclusions make the VAJ closer to the VBR at the level of LCS and NCS, lessening the difference between the internal and external height. Despite these observations having been done in human cadavers studies (29-31), in our experience we have observed that also at the level of the right coronary sinus and of the commissure between the left and the right sinus, a deeper root dissection of the external face of the root is still possible in order to reach the level of the VBR despite the presence of muscular portion of the interventricular septum.

The virtual plane between the VAJ and the right ventricular muscle in most of the cases can be gently dissected and exposed without grossly entering the muscular fibers of the left ventricle. In our experience the presence of the VAJ does not prevent reaching the level of the VBR where the annuloplasty ring or a Dacron graft need to be positioned. However, sometimes, because of the tissue thickness due to the presence of muscle, the external 
circumference of the VBR will result in significantly larger sizing than the internal circumference of the VBR. This aspect needs to be considered when choosing the size of the external ring or band needed to perform an annuloplasty.

Given these discrepancies in tissue thickness the size of the ring might vary. However, irrespective of the ring size used, the amount of annular reduction should be verified by locking the annuloplasty band or ring around an Hegar dilator of known diameter (usually $21-23 \mathrm{~mm}$ for a normal adult). Root dissection is a fundamental step for valvesparing surgery both for annular stabilization and sinuses replacement. An accurate excision of each sinus from the STJ to the VAJ is the first step that allows to correctly scallop them from the commissure to the respective nadir following the crown-like line of the attachment of the aortic leaflets. In this setting, the different characteristics of the external face of the root have a great importance in terms of relationship with the other cardiac chambers and in terms of the different histology of the VAJ.

In particular, for the reimplantation technique, where annular stabilization is one of the main concerns respect the remodeling approach, the presence of muscular fibers of the LVOT into the coronary sinuses that put the VAJ and VBR into different planes, have to be considered. The complete dissection of the external surface of the aortic root should be done not only to properly excise the dilated sinuses of Valsalva and easily suture the valve remnants to the Dacron graft, but also to obtain an effective annular stabilization. Finally, despite several anatomical and conceptual differences, the VAJ and the VBR are strongly correlated $(32,33)$ and should not be considered as separated entities every time an aortic annuloplasty is performed.

As regarding to the maneuvers to modify the leaflet free margin (usually a free margin reduction is needed to reduce the cusp prolapse), we should make use of the known anatomy and histology. In fact, the nodule of arantii, given its fibrotic component is the optimal spot to perform a cusp plication with a reduced risk of cusp damage. Furthermore, this is a zone with reduced stress as compared to the portion close to the commissure where the opening and closing of the leaflet cause a much higher stress on the cusp free margin. In conclusion, the deep knowledge of the root anatomy is the first step towards a satisfactory root reconstruction and accurate aortic valve repair.

\section{Acknowledgments}

None.

\section{Footnote}

Conflicts of Interest: The authors have no conflicts of interest to declare.

\section{References}

1. Cook AC, Anderson RH. Attitudinally correct nomenclature. Heart 2002;87:503-6.

2. Salica A, Pisani G, Morbiducci U, et al. The combined role of sinuses of Valsalva and flow pulsatility improves energy loss of the aortic valve. Eur J Cardiothorac Surg 2016;49:1222-7.

3. Leyh RG, Schmidtke C, Sievers HH, et al. Opening and closing characteristics of the aortic valve after different types of valve preserving surgery. Circulation 1999;100:2153-60.

4. Ho SY. Structure and anatomy of the aortic root. Eur J Echocardiogr 2009;10:13-10.

5. Prêtre R, Kadner A, Dave H, et al. Tricuspidisation of the aortic valve with creation of a crown-like annulus is able to restore a normal valve function in bicuspid aortic valves. Eur J Cardiothorac Surg 2006;29:1001-6.

6. El Khoury G, Glineur D, Rubay J, et al. Functional classification of aortic root/valve abnormalities and their correlation with etiologies and surgical procedures. Curr Opin Cardiol 2005;20:115-21.

7. Silver MA, Roberts WC. Detailed anatomy of the normally functioning aortic valve in hearts of normal and increased weight. Am J Cardiol 1985;55:454-61.

8. Tamás E, Nylander E. Echocardiographic description of the anatomic relations within the normal aortic root. J Heart Valve Dis 2007;16:240-6.

9. Anderson RH. The surgical anatomy of the aortic root. Multimed Man Cardiothorac Surg 2007;2007:mmcts.2006.002527.

10. Anderson RH, Devine W, Ho SY, et al. The myth of the aortic annulus: the anatomy of the subaortic outflow tract. Ann Thorac Surg 1991;52:640-6.

11. de Kerchove L, El Koury G. Anatomy and pathophysiology of the ventriculo-aortic junction: implication in aortic valve repaire surgery. Ann Cardiothorac Surg 2013;2:57-64.

12. Blanke P, Russe M, Leipsic J, et al. Conformational pulsatile changes of the aortic annulus: impact on prosthesis sizing by computed tomography for transcatheter aortic valve replacement. JACC Cardiovasc Interv 2012;5:984-94. 
13. Chirichilli I, Irace F, Weltert L, et al. Morphological modification of the aortic annulus in tricuspid and bicuspid valves after aortic valve reimplantation procedure: an electrocardiography-gated computed tomography study. Eur J Cardiothorac Surg 2019. (In Press).

14. Mori S, Anderson RH, Tahara N, et al. The differences between bisecting and off-center cuts of the aortic root: the three-dimensional anatomy of the aortic root reconstructed from the living heart. Echocardiography 2017;34:453-61.

15. Berdajs D, Lajos $P$, Turina $M$. The anatomy of the aortic root. Cardiovasc Surg 2002;10:320-7.

16. Choo SJ, McRae G, Olomon JP, et al. Aortic root geometry: pattern of differences between leaflets and sinuses of Valsalva. J Heart Valve Dis 1999;8:407-15.

17. Thubrikar M, Bosher LP, Nolan SP. The mechanism of opening of the aortic valve. J Thorac Cardiovasc Surg 1979;77:863-70.

18. Thubrikar MJ, Nolan SP, Aoud J, et al. Stress sharing between the sinuses and leaflets of canine aortic valve. Ann Thorac Surg 1986;42:434-40.

19. De Hart J, Peters GW, Shreurs PJ, et al. A threedimensional computational analysis of fluid-structure interaction in the aortic valve. J Biomech 2003;36:103-12.

20. Morbiducci U, Ponzini R, Rizzo G, et al. Mechanistic insight into the physiological relevance of helical blood flow in the human aorta. An in vivo study. Biomech Model Mechanobiol 2011;10:339-55.

21. Kvitting JP, Ebbers T, Wingström L, et al. Flow patterns in the aortic root and the aorta studied with time-resolved, 3-dimensional, phase contrast magnetic resonance imaging: implication for aortic valve-sparing surgery. J Thorac Cardiovasc Surg 2004;127:1602-7.

22. Higashidate $M$, Tamiya K, Beppu T, et al. Regulation of the aortic valve opening. J Thorac Cardiovasc Surg

Cite this article as: De Paulis R, Salica A. Surgical anatomy of the aortic valve and root-implications for valve repair. Ann Cardiothorac Surg 2019;8(3):313-321. doi: 10.21037/ acs.2019.04.16
1995;110:496-503.

23. Bellhouse BJ, Bellhouse FH. Mechanism of closure of the aortic valve. Nature 1968;217:86-7.

24. Pisani G, Scaffa R, Ieropoli O, et al. Role of the sinuses of Valsalva on the opening of the aortic valve. J Thorac Cardiovasc Surg 2013;145:999-1003.

25. Yoganathan AP, Cape EG, Sung HW, et al. Review of hydrodynamic principles for the cardiologist: applications to the study of blood flow and jets by imaging techniques. J Am Coll Cardiol 1988;12:1344-53.

26. Yellin EL, Peskin CS. Large amplitude pulsatile water flow across an orifice. J Dyn Sys Meas Control 1975;97:92-5.

27. Grande-Allen KJ, Cochran RP, Reinhall PG, et al. Recreation of sinuses is important for sparing the aortic valve: a finite element study. J Thorac Cardiovasc Surg. 2000;119:753-63.

28. Weltert L, De Paulis R, Scaffa R, et al. Re-creation of a sinuslike graft expansion in Bentall procedure reduces stress at the coronary button anastomoses: a finite element study. J Thorac Cardiovasc Surg 2009;137:1082-7.

29. de Kerchove L, Jashari R, Boodhwani M, et al. Surgical anatomy of the aortic root: implication for valve-sparing reimplantation and aortic valve annuloplasty. J Thorac Cardiovasc Surg 2015;149:425-33.

30. Khelil N, Sleilaty G, Palladino M, et al. Surgical anatomy of the aortic annulus: landmarks for external annuloplasty In aortic valve repair. Ann Thorac Surg 2015;99:1220-6.

31. Lansac E, de Kerchove L. Aortic valve repair techniques: state of the art. Eur J Cardiothorac Surg 2018;53:1101-7.

32. Anderson RH, Mori S. Nomenclature of the components of the aortic root. Eur J Cardiothorac Surg 2018. [Epub ahead of print].

33. Lansac E, de Kerchove L. Reply to Anderson and Mori. Eur J Cardiothorac Surg 2018. [Epub ahead of print]. 